\title{
Therapeutic approaches and drug-resistance in chronic lymphocytic leukaemia
}

\author{
Narjis Fatima ${ }^{1,2}$, Kyle R. Crassini ${ }^{1}$, Lauren Thurgood ${ }^{3}$, Yandong Shen ${ }^{1,2}$, Richard I. Christopherson ${ }^{2}$, \\ Bryone Kuss $^{3}$, Stephen P. Mulligan ${ }^{1,2}$, Oliver Giles Best ${ }^{1,2,3}$ \\ 'Kolling Institute of Medical Research, University of Sydney, Royal North Shore Hospital, Sydney, NSW 2065, Australia. \\ ${ }^{2}$ School of Life and Environmental Sciences, University of Sydney, Sydney NSW 2008, Australia. \\ ${ }^{3}$ College of Medicine and Public Health, Flinders University, Bedford Park, Adelaide, SA 5042, Australia.
}

Correspondence to: Dr. Oliver Giles Best, College of Medicine and Public Health, Flinders University, Bedford Park, Adelaide, SA 5042, Australia. E-mail: giles.best@flinders.edu.au

\begin{abstract}
How to cite this article: Fatima N, Crassini KR, Thurgood L, Shen Y, Christopherson RI, Kuss B, Mulligan SP, Best OG. Therapeutic approaches and drug-resistance in chronic lymphocytic leukaemia. Cancer Drug Resist2020;3:532-49.

http://dx.doi.org/10.20517/cdr.2019.111
\end{abstract}

Received: 4 Dec 2019 First Decision: 5 Mar 2020 Revised: 24 Mar 2020 Accepted: 31 Mar 2020 Available online: 11 May 2020

Science Editor: Lee M. Graves Copy Editor: Jing-Wen Zhang Production Editor: Jing Yu

\begin{abstract}
The treatment of chronic lymphocytic leukaemia has been revolutionised in recent years, first by the introduction of chemoimmunotherapy regimens and subsequently by the development of drugs, including ibrutinib, idelalisib and venetoclax, that target components of the B-cell receptor signalling pathway or B-cell lymphoma 2 family of proteins. Despite high initial response rates in patients treated with chemoimmunotherapy or targeted agents, a significant proportion of patients relapse with progressive and refractory disease. In a subset of these patients, drug resistance has been associated with specific genetic lesions or activation of alternate pro-survival pathways. However, the mechanisms that confer drug resistance in the remainder of the patients with refractory disease have yet to be fully elucidated. In this review, we discuss our current understanding of the mechanics of drug resistance in chronic lymphocytic leukaemia and describe how this knowledge may aid in rationalising future treatment strategies to prevent the development of refractory or aggressive transformation of the disease.
\end{abstract}

Keywords: Chronic lymphocytic leukaemia, drug resistance, targeted cancer therapy

\section{INTRODUCTION}

Chronic lymphocytic leukaemia (CLL) is the most common leukaemia among adults in the Western world. The disease is characterised by the accumulation of apoptosis-resistant B lymphocytes in the blood, 
bone marrow, lymph nodes and spleen. The bone marrow and lymph nodes play a significant role in the pathogenesis and drug sensitivity of CLL. Within these tissues the leukaemic cells interact with a variety of cells, including stromal cells, $\mathrm{T}$ cells and adipocytes, forming pseudofollicular structures known as proliferation centres. CLL cells within proliferation centres are phenotypically distinct from circulating CLL cells with antigen expression changes consistent with B cell activation and proliferation ${ }^{[1]}$.

Despite significant advances in the treatment of CLL in recent years, the disease is still widely considered incurable. In many patients, drug resistance, relapse and disease progression or aggressive transformation have been associated with specific genetic lesions. The most well described of these lesions is deletion of the region on the short arm of chromosome 17 (del17p), which encompasses the TP53 gene. Under normal cellular conditions, expression of the TP53 protein is low but is induced by DNA damage, resulting in a signalling cascade that triggers cell cycle arrest and DNA repair or apoptosis. The importance of TP53 in cancer is highlighted by the fact that over $50 \%$ of all cancers harbour mutations in the TP53 gene. In CLL, as in other cancers, TP53 lesions are strongly associated with poor outcome following many of the current therapeutic strategies.

Deletions or mutations of the ataxia telengiectasia mutated (ATM) gene are also common among CLL patients and are associated with intermediate risk disease ${ }^{[2,3]}$. ATM is upstream of TP53 in signalling the response of cells to DNA damage, acting as a "sensor" of DNA breaks. Following DNA damage, ATM is activated and phosphorylates the ubiquitin ligase MDM-2, which dissociates from TP53. This stabilises the TP53 protein enabling it to translocate from the cytoplasm into the nucleus where it functions as a transcription factor regulating the expression of multiple pro-apoptotic or cell cycle regulators. Deletions of TP53 or ATM, in the 17p13 and 11q22-q23 chromosomal regions, respectively, are identified in approximately $4 \%-10 \%$ (TP53) and 10\%-20\% (ATM) of patients at diagnosis, but the proportion increases to around $40 \%\left(T_{53}\right)$ in patients with relapsed/refractory disease ${ }^{[4]}$. Earlier studies suggested that fludarabine-based chemoimmunotherapy (CIT) regimens may overcome the poor prognosis of patients with ATM deletions ${ }^{[5]}$; however, the German CLL8 trial $^{[6]}$ and a recent study suggests that these patients have significantly shorter progression-free survival duration ${ }^{[7]}$.

A better understanding of the mechanisms that drive CLL-cell survival and proliferation, particularly within the tumour microenvironment, has led to the development of novel, more targeted therapies. However, as data from trials of these newer agents accumulate, it has become increasingly evident that a significant proportion of patients still relapse with progressive disease. High throughput genetic sequencing techniques, such as next-generation sequencing, have led to a rapid increase in our understanding of the genetic diversity of CLL. The ability to monitor patients from diagnosis through treatment to relapse have shown the marked clonal evolution that occurs in $\mathrm{CLL}^{[8]}$. As highlighted, TP53 lesions are shown to occur more frequently in a relapsed and refractory setting and remain strong predictors of poor outcome. However, more sensitive sequencing techniques have shown that exposure to novel therapeutic agents can result in the emergence of clones harbouring additional molecular lesions, many of which may be associated with drug resistance. Identifying effective therapeutic approaches, particularly for poor-risk disease patients, remains an important area of research for CLL. Elucidating the mechanisms of drug resistance in CLL is key in the development of novel therapeutic approaches that will improve patient outcomes and mitigate the risk of drug resistance.

This review discusses the significant advances in CLL therapy that have occurred in the last two decades and describes our current understanding of the mechanisms of drug resistance in this disease.

\section{CIT}

CIT refers to therapy regimens incorporating both chemotherapeutic drugs and antibodies that bind to specific antigens on cancer cells. The development of anti-CD20 antibodies and incorporation of these into 
chemotherapy regimens represented a significant milestone in the treatment of $\mathrm{CLL}^{[9,10]}$. CIT regimens are associated with significantly higher response rates compared to chemotherapy-only regimens ${ }^{\left[10^{-12}\right]}$ and CIT remains a commonly used front-line treatment option for CLL.

Fludarabine and cyclophosphamide in combination with the anti-CD20 monoclonal antibody rituximab (FCR) remains the most widely utilised of the CIT regimens in CLL; initial trials demonstrated that the addition of rituximab to the FC regimen was associated with a significant improvement in both progression-free survival and overall survival. FCR has proven to be an effective therapy for many patients, particularly those with mutated $I G H V$, with half of these patients remaining in permanent complete remission ${ }^{[13]}$. However, FCR therapy is commonly associated with neutropenia, an increased risk of infection and an increased risk of treatment-related myelodysplasia and acute myeloid leukaemia ${ }^{[14]}$.

The mechanisms of action of rituximab have been reported to include antibody-dependent cellular cytotoxicity (ADCC), complement-dependent cellular cytotoxicity (CDC), chemo-sensitisation, direct cytotoxicity and antibody dependent cellular phagocytosis, although the contribution of these effects to the overall efficacy of rituximab appear to be disease dependent. There are direct effects of rituximab on the translocation of the CD20 molecule in the plasma membrane-associated lipid rafts, which results in increased calcium flux and caspase-dependent induction of apoptosis ${ }^{[15]}$. We and others have confirmed that this redistribution occurs in CLL cells following treatment with rituximab ${ }^{[16,17]}$.

Resistance to CIT in CLL arises due to the presence or acquisition of a specific lesion(s) that is present in a subclone of CLL cells rendering these cells insensitive to therapy. During or after therapy, evolution or selection of these resistant clones occurs. Such lesions commonly arise in the DNA damage-response pathway, in the ATM and TP53 genes. Patients with TP53 lesions of in particular generally respond poorly to FCR or relapse early with aggressive disease following treatment ${ }^{[3,6,18]}$. This occurs largely because the efficacy of the FCR regimen is dependent on the ability of the leukaemic cells to mount a DNA-damage response, which under normal circumstances triggers apoptosis or cell cycle arrest. In patients with lesions in ATM or TP53, DNA damage induced by these drugs does not trigger an apoptotic signalling cascade or block cell proliferation. While the prognostic significance of high-frequency deletions or mutations of TP53 is well established, the impact of low frequency lesions remains unclear. In a recent study of newly diagnosed CLL patients, Brieghel et al. ${ }^{[19]}$ found that the presence of TP53 mutations with a variant allele frequency of $>10 \%$ in the absence of TP53 deletion had no impact on overall survival or treatment-free survival. However, in patients requiring treatment, the presence of TP53 mutations with variant allele frequencies of $>1 \%$ were of prognostic significance. As the authors suggested, these data indicated the need for further studies to define the level at which TP53 mutations are clinically significant.

Several studies have identified associations between resistance to fludarabine and rituximab and microRNA (miR) expression in CLL cells. Resistance to fludarabine has been linked to increased expression of miRs148a, 222 and $21^{[20]}$ and rituximab resistance has been associated with decreased expression of miRs-125b and $532-3 \mathrm{p}^{[21]}$. While many miRs are regulated by TP53, Cerna et al ${ }^{[22]}$ demonstrated that expression of miR-34a has independent prognostic significance, suggesting that low expression of miR-34a may confer resistance to FCR in a subset of patients with no lesion of either ATM or TP53. The same study also showed that increased miR-34a expression following FCR-induced DNA damage represses the transcriptional activity of FOXP1 and results in a reduction of $\mathrm{B}$ cell receptor (BCR)-mediated signalling. This suggests that while CIT regimens do not directly inhibit intracellular signalling, the efficacy of FCR in CLL may depend on indirect inhibition of kinases in the BCR pathway.

Next-generation sequencing techniques have enabled detailed examination of the genome of CLL cells and have highlighted a number of high frequency genetic variants including NOTCH1, SF3B1 and BIRC3. 
Mutations of $\mathrm{NOTCH}_{1}$ have been associated with decreased levels of $\mathrm{CD} 20$ via a mechanism involving histone deacetylase-mediated repression of $\mathrm{CD} 20$ expression, resulting in reduced sensitivity to CD20targeted therapies in vitro ${ }^{[23]}$. Furthermore, patients with mutations of NOTCH1 received no benefit from addition of rituximab to FC in the CLL8 trial and a weak benefit from addition of ofatumumab to chlorambucil in the phase III trial COMPLEMENT ${ }^{[24]}$, suggesting that mutations of NOTCH1 may confer reduced sensitivity or resistance to therapy with anti-CD20 monoclonal antibodies.

Longitudinal studies on CLL patients who relapse following FCR treatment suggest that clonal selection and evolution both occur. Clonal selection occurs when lesions are evident, often as minor clones, prior to treatment but predominate following treatment. Clonal evolution differs in that lesions are not present prior to therapy but are induced in cells by the genotoxic nature of the treatment. Genetic damage induced by treatment may subsequently confer a survival advantage and trigger the propagation of a new, more aggressive, disease clone. This was demonstrated in an analysis of patients enrolled in the German CLL8 trial, in which copy number changes were twice as likely following treatment with FC or FCR than over a similar period prior to treatment ${ }^{[25]}$. Studies have also demonstrated that accumulation of genetic aberrations and increased genetic complexity resulting from chromosomal instability are associated with poor outcome and response to treatment ${ }^{[26,27]}$.

Trials of other CIT regimens, including bendamustine in combination with rituximab (BR) demonstrate this is also an effective and well-tolerated treatment regimen, even among elderly patients, with an overall response rate of $45.5 \%{ }^{[28]}$. Treatment with BR is associated with a significantly lower incidence of neutropenia than FCR (19.7\% in BR-treated compared to 34\% in FCR-treated patients). However, unmutated $I G H V$ gene status and/or deletions of chromosome 17p remained strong indicators of poor response or outcome following treatment with BR. Furthermore, unlike FCR, there is no plateau in the survival curve of $I G H V$-mutated patients treated with BR. Similar to fludarabine, patients with deletions or mutations of TP53, have poor outcomes following treatment with bendamustine due to the dependence of the drug on the TP53-mediated apoptotic cascade ${ }^{[28]}$. Factors associated with resistance to the FCR and BR regimens are detailed in Table 1.

Next-generation anti-CD20 monoclonal antibodies have also been developed to optimise the binding and cytotoxic effects towards B cells. Ofatumumab is a fully humanized, type I, CD20-specific monoclonal antibody. Ofatumumab binds to CD20 with greater affinity than does rituximab and has a slower dissociation rate ${ }^{[29]}$. Although, ofatumumab does not induce direct cell toxicity, the greater avidity of the antibody for CD20 means it is less affected by complement regulatory proteins and has greater CDCmediated effects compared to rituximab ${ }^{[30]}$.

Obinutuzumab is a type II, anti-CD20 monoclonal antibody that has a distinct variable region and recognises a different CD20 epitope compared to rituximab. The high affinity of obinutuzumab for CD20 means it is a more potent inducer of direct cell death and ADCC than is rituximab. However, obinutuzumab is a less potent inducer of $\mathrm{CDC}$, since the obinutuzumab-CD20 complex is unable to move into lipid rafts. Obinutuzumab has been shown to have activity in relapsed/refractory CLL patients ${ }^{[31]}$. In the German CLL study group (GCLLSG) CLL11 trial, patients with comorbidities were treated with chlorambucil alone, chlorambucil plus obinutuzumab or chlorambucil plus rituximab. This trial demonstrated that both the response and minimal residual disease (MRD)-negativity rate of patients treated with chlorambucil plus obinutuzumab were significantly higher than those of patients in the other arms. In terms of resistance related to $\mathrm{NOTCH} 1$ mutations, as stated, ofatumumab appears to be similar to rituximab; however, there is some evidence to suggest that this resistance is overcome by obinutuzumab ${ }^{[32,33]}$. In the current era, treatment refractoriness or resistance to CIT modalities in CLL is most commonly addressed by a change in therapeutic approach to targeted therapies. 
Table 1. Details of the features associated with resistance or poor response of CLL patients to current therapeutic drugs or regimens

\begin{tabular}{|c|c|c|c|}
\hline Drug/Regimen & Drug target(s) & $\begin{array}{c}\text { Features independently associated with resistance or poor } \\
\text { response to treatment }\end{array}$ & Ref. \\
\hline \multirow[t]{8}{*}{ FCR } & \multirow{8}{*}{$\begin{array}{l}\text { Genotoxic and anti-CD20 } \\
\text { monoclonal antibody }\end{array}$} & Deletion of 17p13 (TP53) & {$[3,18]$} \\
\hline & & Deletion of 11q22-23 (ATM) & {$[3,7]$} \\
\hline & & TP53 or ATM mutations & {$[6,7]$} \\
\hline & & NOTCHI, BIRC3, SF3B1, RPS15 mutations & {$[6,119]$} \\
\hline & & Increased expression of miRs 148a, 222 and 21 (fludarabine) & [20] \\
\hline & & Decreased expression of miRs $125 b$ and $532-3 p$ (rituximab) & [21] \\
\hline & & Low miR34a expression & {$[22]$} \\
\hline & & Unmutated /gHV status & {$[13,18,120]$} \\
\hline \multirow[t]{3}{*}{ BR } & \multirow{3}{*}{$\begin{array}{l}\text { Genotoxic and anti-CD20 } \\
\text { monoclonal antibody }\end{array}$} & Deletion of 17p13 (TP53) & {$[121]$} \\
\hline & & Deletion of 11q22-23 (ATM) & [7] \\
\hline & & Unmutated /gHV status & [122] \\
\hline \multirow[t]{6}{*}{ Ibrutinib } & \multirow[t]{6}{*}{ BTK } & BTK mutations (C481 or V5371) & {$[46,47]$} \\
\hline & & $P L C-g 2$ mutations & {$[46]$} \\
\hline & & PD-1 overexpression & {$[50]$} \\
\hline & & Pro-survival shift in BCL-2 protein expression & {$[50,52]$} \\
\hline & & Increased AKT/mTOR signalling & {$[50]$} \\
\hline & & Deletion of $8 p$ and decreased TRAIL-R expression & {$[123]$} \\
\hline Acalabrutinib & BTK & BTK mutations (C481) & [47] \\
\hline \multirow[t]{6}{*}{ Venetoclax } & \multirow[t]{6}{*}{$\mathrm{BCL} 2$} & $B C L 2$ mutations & {$[90,91]$} \\
\hline & & Mutations in $B T G 1$ and $B R A F$ & [89] \\
\hline & & Deletion of CDNK2A/B & [89] \\
\hline & & Amplification of PD-L1 & [89] \\
\hline & & MCL1 overexpression & [108] \\
\hline & & Mitochondrial reprogramming & [93] \\
\hline \multirow[t]{2}{*}{ Idelalisib } & \multirow[t]{2}{*}{ PI3-kinase-d } & Increased IGF1R expression and MAPK-ERK1/2 activity & [68] \\
\hline & & Mutations in MAPK-ERK1/2 pathway (KRAS, BRAF, MAP2K1) & [69] \\
\hline
\end{tabular}

BTK: Bruton's tyrosine kinase; FCR: fludarabine, cyclophosphamide, rituximab; BR: bendamustine, rituximab; ATM: ataxia telengiectasia mutated; MAPK: mitogen-sctivated protein kinase

\section{INHIBITORS OF BCR-MEDIATED SIGNALLING}

Tonic activation of the BCR signalling pathway is a hallmark of CLL cells and plays a key role in their survival and proliferation. Signalling via the BCR is activated through binding of specific antigens to the receptor and as a result of the interaction of CLL cells with a variety of other cell types, including stromal cells and $\mathrm{T}$ cells ${ }^{[34]}$. The BCR pathway also plays a crucial role in mediating the effects of a variety of chemokines and growth factors. Signalling via the BCR is mediated by a range of receptor-associated and intracellular kinases, which ultimately induce a protein expression profile consistent with B cell survival, proliferation, migration and adhesion ${ }^{[35]}$. A better understanding of the important roles of the BCR pathway and the tumour microenvironment in CLL has driven the clinical development of drugs that inhibit key signalling components within the BCR pathway. Table 1 and the schematic diagram, Figure 1, detail and illustrate the mechanisms of action and drug resistance associated with the therapeutic agents used in CLL to target the BCR signalling pathway and inhibit B-cell lymphoma 2 (BCL2).

A phenomenon common to the drugs described here is the rapid lymphocytosis observed in most patients following commencement of therapy. This effect is interesting from a biological perspective since it highlights the mechanisms by which CLL cells migrate to and are retained within the tumour microenvironment; BCR-targeted drugs alter the expression of chemokine receptors including CXCR $4^{[36,37]}$ and adhesion molecules including $\mathrm{CD} 49 \mathrm{~d}^{[38]}$. The redistribution of leukemic cells from the lymph nodes is observed clinically as a rapid decrease in lymph node size with a concomitant rise in the circulating white cell count. In most patients this lymphocytosis is transient, since the circulating CLL cells no longer receive the support and proliferative drive conferred by the tumour microenvironment. The CLL tumour 


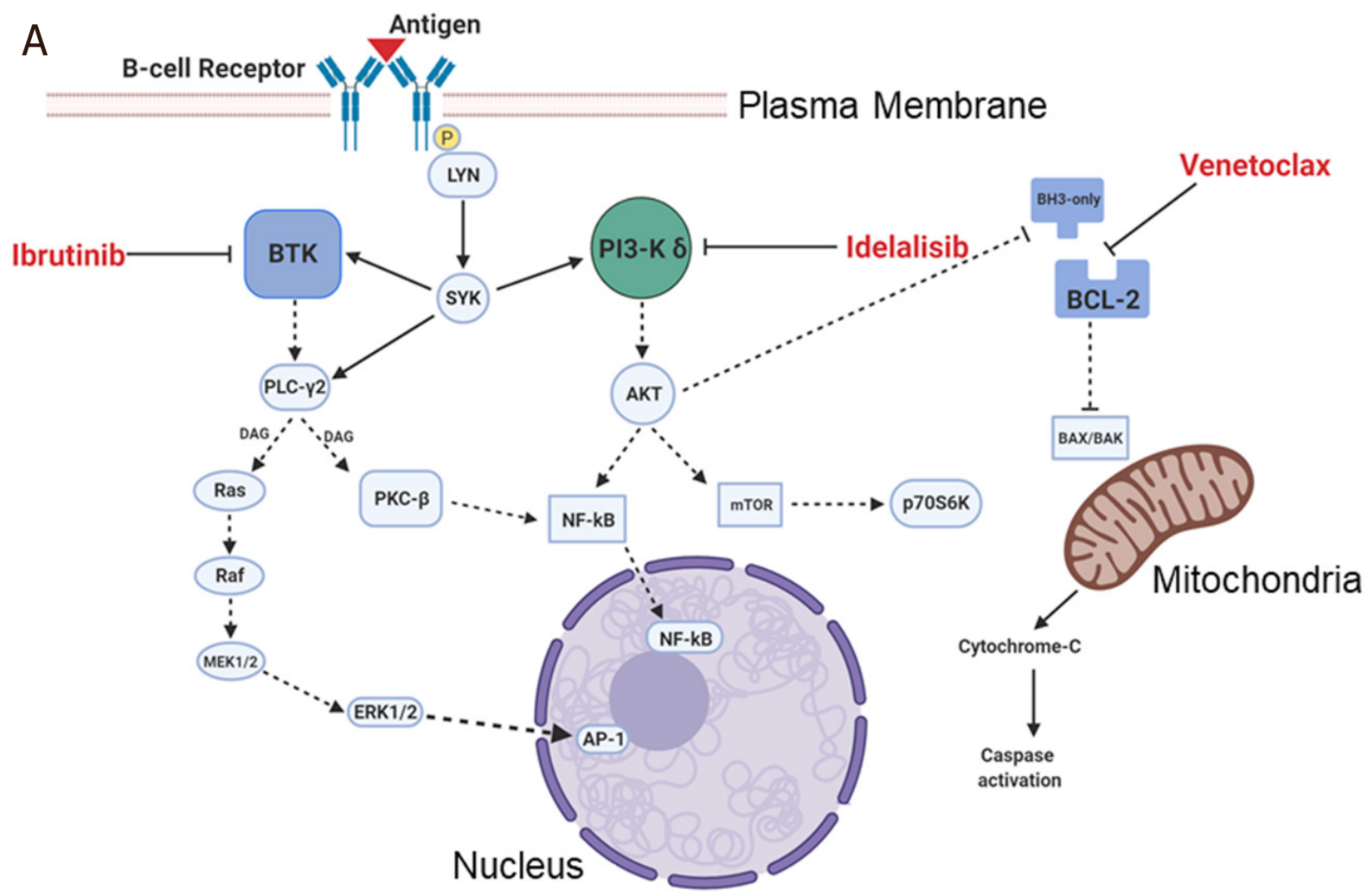

B

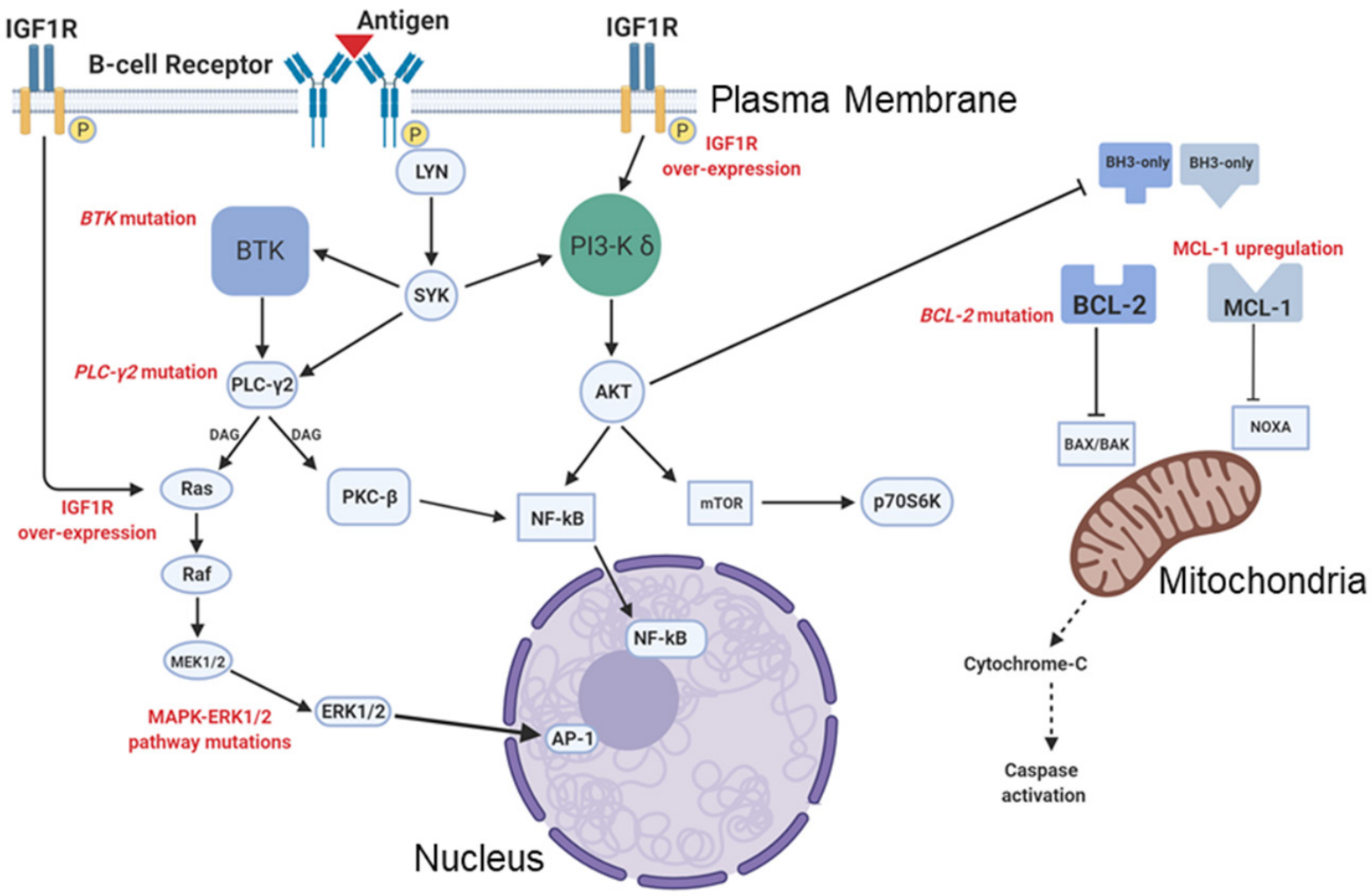

Figure 1. Schematic diagram illustrating the signalling pathways targeted by ibrutinib, idelalisib and venetoclax in CLL (A) and the known mechanisms of resistance to each of these agents (B) in CLL cells. CLL: chronic lymphocytic leukaemia; Btk: bruton's tyrosine kinase; LYN: Lck/Yes novel tyrosine kinase; AKT: protein kinase B; SYK: spleen tyrosine kinase; PKC: protein kinase C; BCL: B-cell Lymphoma; MCL-1: myeloid leukemia cell differentiation protein; mTOR: mammalian target of rapamycin 
microenvironment may also play an important role in mediating resistance or reduced sensitivity to the BCR-targeted therapies discussed in the following section. The interaction of CLL cells with other cells present in the tumour microenvironment and certain cytokines and growth factors stimulate intracellular signalling pathways that promote CLL cell survival and counter the efficacy of targeted therapies. This is illustrated in a recent study by Kapoor et al. ${ }^{[39]}$, in which reduced sensitivity of CLL and diffuse large B-cell lymphoma (DLBCL) cells to ibrutinib was associated with increased activity of the PI3-kinase pathway.

\section{INHIBITORS OF BRUTON'S TYROSINE KINASE}

\section{Ibrutinib}

Ibrutinib is an irreversible inhibitor of Bruton's tyrosine kinase (BTK). BTK is a non-receptor tyrosine kinase, which is constitutively active in CLL cells as a result of tonic BCR-mediated signalling and activity of the kinases Lck/Yes novel tyrosine kinase (LYN) and spleen tyrosine kinase (SYK). Ibrutinib binds at the cysteine 481 (C481) residue within the kinase domain of BTK and prevents the phosphorylation of its downstream targets ${ }^{[40]}$.

As a single agent, ibrutinib has proven highly effective, initially in the treatment of relapsed/refractory CLL patients and more recently as a frontline therapy ${ }^{[41,42]}$. Adverse events, including atrial fibrillation, excessive bleeding, and arthralgia are common among CLL patients treated with ibrutinib ${ }^{[43,44]}$.

Despite high initial response rates to ibrutinib, a significant proportion of patients relapse with progressive disease. Treatment options for patients with disease transformation are very limited. Resistance to ibrutinib is more common among heavily pre-treated patients and is associated with mutations of the C481 residue in BTK, which permits only reversible binding of ibrutinib to the kinase ${ }^{[45,46]}$. Mutations of phospholipase-g2 (PLC-g2) that allow the circumvention of BTK and activation of signalling of the same pathway have also been associated with resistance to ibrutinib ${ }^{[46]}$. Similar to mutations of the C481 residue, the V537I, T474A and L528W BTK mutations identified in ibrutinib-resistant patients are all located within the kinase domain and are believed to interfere with the ability of ibrutinib to bind to the protein ${ }^{[47]}$. The T316A mutation documented in a single ibrutinib-resistant patient is distinct since it is located within the Src homology 2 ( $\mathrm{SH}_{2}$ ) domain of BTK and confers resistance to ibrutinib by modifying intracellular signalling pathways rather than by interfering with ibrutinib binding ${ }^{[48]}$. The study proposed that mutations within the $\mathrm{SH} 2$ domain may interfere with the ability of BTK to interact with key intermediary proteins and to phosphorylate $P L C$-g2. In all these studies the BTK mutations were not evident prior to the patients developing ibrutinib-resistant disease, although another study demonstrated that BTK mutations frequently predate clinical resistance ${ }^{[49]}$. Collectively, the studies highlight the association between mutations in $B T K$ or $P L C-g 2$ and ibrutinib resistance and reinforce the importance of this signalling pathway in the pathogenesis of CLL.

A relatively small proportion of patients develop ibrutinib resistance in the absence of detectable mutations of either BTK or PLC-g2. Although the exact mechanisms of resistance in these cases remain unclear, it can be speculated that mutations in signalling components downstream of BTK, in the mitogen-sctivated protein kinase (MAPK) pathway, for example, or dysregulation of another pro-survival pathway may also confer resistance to ibrutinib in CLL. This notion is supported by a study in which overexpression of programmed cell death protein-1 (PD-1) and BCL2 and increased activity of the AKT/mTOR pathway were observed in CLL patients who developed progressive disease or Richter's transformation following treatment with ibrutinib ${ }^{[50]}$. Jain et al..$^{[51]}$, demonstrated a similar mechanism in ibrutinib-resistant DLBCL cell lines and showed that survival of these lines was dependent on signalling via the phosphoinositol 3 kinase (PI 3-kinase) pathway. Since proteins of the BCL2 family are regulated by the activity of the PI3kinase/AKT pathway, it is conceivable that increased PI3-kinase activity in CLL cells may account for the pro-survival shift in expression of the BAX and BCL2 proteins, which correlates with poor outcome 
following ibrutinib treatment ${ }^{[52]}$. Since the mechanisms of action of ibrutinib include downregulation of integrin expression, which liberates CLL cells from the tumour microenvironment, the expression of proteins involved in the retention of leukaemic cells within the lymph nodes may play a significant role in the response of patients to this drug. This was illustrated in the study by Tissino et al. ${ }^{[53]}$, in which expression of CD49d and activation of the integrin VLA-4 were associated with reduced nodal clearance and shortened progression-free survival in patients treated with ibrutinib. Irrespective of the mechanism, the fact that a significant proportion of patients develop ibrutinib-resistant disease underscores the importance of on-going trials of ibrutinib in combination with other agents, including venetoclax ${ }^{[54]}$ and umbralisib $^{[55]}$.

\section{Next-generation BTK inhibitors}

Trials of the next-generation BTK inhibitors acalabrutinib ${ }^{[56]}$, zanubrutinib $^{[57]}$, tirabrutinib ${ }^{[58]}$ (NCT02983617, NCT02457598) and LOXO-305 (NCT03740529) are currently underway as single agents or in combinations for haematological diseases. In addition, efficacy of the BTK inhibitors evobrutinib ${ }^{[59]}, \mathrm{ABBV}-105^{[60]}$ and poseltinib $^{[61]}$ has been assessed for multiple sclerosis, systemic lupus erythematosus and auto-immune diseases, respectively. Agents that selectively inhibit both BTK and other kinases have also been developed. Vecabrutinib is a reversible inhibitor of BTK that also targets interleukin-2-inducible tyrosine kinase while CG-806 is an inhibitor of both BTK and the FMS-like tyrosine kinase-3 (FLT-3). ARQ-531 inhibits BTK as well as a range of other kinases including LYN and MEK1. Many of these next-generation BTK inhibitors are reported to share the same mechanism of action as ibrutinib but were designed to be more specific for BTK with less effect against other members of the TEC family of kinases compared to ibrutinib. A study describing the biochemical effects of ibrutinib compared to those of tirabrutinib, acalabrutinib and spebrutinib has recently been published ${ }^{[62]}$. Many of these drugs, including the reversible BTK inhibitors LOXO-305, vecabrutinib and ARQ-531, also inhibit both wild-type and BTK harbouring mutations of the C481 residue. No data are available yet concerning whether these drugs are associated with a lower risk of bleeding or rates of atrial fibrillation, which are the main complications associated with ibrutinib ${ }^{[63]}$.

Currently, the limited follow-up data from trials of CLL patients treated with next-generation BTK inhibitors also means it is too early to determine the incidence of drug resistance among these patients. Acalabrutinib is a more potent and selective BTK inhibitor than ibrutinib and is believed to be associated with a lower rate of adverse events. The first published study reporting resistance among CLL patients treated with acalabrutinib identified 2 patients, one of whom developed resistant disease with a mutation in BTK, the other who developed a Richter's transformation with a mutation in BTK and a dominant TP53mutated clone ${ }^{[47]}$. The patients who developed progressive disease and a Richter's transformation while on treatment with acalabrutinib both had mutations of the C481 residue in BTK. Given that the allele frequency of BTK and PLC-g2 mutations is often low in BTK inhibitor-resistant patients, it seems likely that these mutations are only part of the story and that other genetic lesions or pro-survival mechanisms play a significant role in the resistance of patients to these drugg ${ }^{[6]]}$.

\section{PI3-KINASE INHIBITORS}

\section{Idelalisib}

Idelalisib is a specific inhibitor of the d-isoform of phosphoinositide 3'-kinase (PI3-kinase). All the PI3kinase isoforms, a, b, g and d, mediate signals downstream of the BCR and play important roles in the survival and proliferation of CLL cells. However, the PI3K-d isoform is the predominant isoform expressed in hematopoietic cells and targeting this isoform has proven highly effective in CLL particularly for patients with relapsed/refractory disease ${ }^{[65]}$.

Despite promising initial results ${ }^{[6]}$, further clinical development of idelalisib has been limited by its high adverse event rate, most notably in the form of auto-immune-mediated colitis ${ }^{[66]}$. There are also a limited 
number of studies that have explored mechanisms of idelalisib-resistance in CLL (reviewed in study ${ }^{[67]}$ ). Although the exact mechanisms are unclear, a study of a CLL mouse model and primary CLL cells in vitro suggested that, while resistance to idelalisib is not associated with specific gene mutations, it may be linked to increased expression of insulin-like growth factor 1 receptor (IGF1R) and subsequent activation of the MAPK pathway ${ }^{[68]}$. The importance of increased IGF1R expression in this context was confirmed in the same study by demonstrating that idelalisib-resistant CLL cells were sensitive to a specific IGF1R inhibitor. A study by Murali et al ${ }^{[69]}$ also demonstrated the association between increased MAPK-ERK1/2 signalling and resistance of CLL cells to PI3-kinase inhibition. However, in this study of primary CLL cells from idelalisib-resistant patients, the authors showed that drug resistance and constitutive MAPK pathway activation was associated with the acquisition of recurrent mutations in the KRAS, BRAF and MAP2K1 genes $^{[69]}$. Evidence of crosstalk between the MAPK and PI3-kinase signalling pathways was also evident in a study from our laboratory, which demonstrated that inhibition of MAPK-ERK1/2 signalling resulted in increased phosphorylation of AKT in primary CLL cells ${ }^{[70]}$. Under these conditions, it appeared that any cytotoxic effects of the MEK1/2 inhibitor binimetinib were countered by an increase in the phosphorylation of AKT. The ramifications of this are discussed in more detail in the later section on future therapeutic strategies for CLL.

\section{Duvelisib}

Duvelisib is a specific inhibitor of both the $\mathrm{g}$ and the $\mathrm{d}$ isoforms of PI3-kinase. In the DUO phase III clinical trial, 313 patients with relapsed/refractory CLL were treated with either single agent duvelisib or ofatumumab $^{[71]}$. The study demonstrated that duvelisib was effective as a treatment for relapsed/refractory CLL, even in patients with deletion of $17 \mathrm{p}$, and is significantly more effective than ofatumumab ${ }^{[71]}$. However, due to the limited availability of clinical trial and follow-up data, there have been no published reports concerning duvelisib resistance among CLL patients.

It is conceivable that similar mechanisms that confer resistance to idelalisib may also be pertinent to duvelisib; constitutive activation of MAPK-ERK1/2 signalling, whether through increased phosphorylation of components of this pathway or activating mutations, may compensate for inhibition of PI3-kinasemediated signalling and promote the survival of CLL cells. In a study of B- and T-cell lymphoma, resistance of cell lines to duvelisib and the pan-PI3-kinase inhibitor copanlisib was associated with increased expression of interleukin 6 , which resulted in increased phosphorylation of several intracellular signalling molecules, including STAT, AKT, p70S6K, MAPK and NF- $\mathrm{BB}^{[72]}$. It remains likely that in CLL, resistance to specific inhibitors of PI3-kinase-mediated signalling may also be mediated by as yet undescribed mechanisms that involve compensatory upregulation of alternate pro-survival pathways.

\section{Umbralisib}

Single-agent trials of the dual PI3-kinase-d and casein kinase-1e (CK-1e) inhibitor umbralisib, either alone or in combination with other drugs are also being conducted for CLL. In a phase I study of umbralisib in 90 patients with CLL, B-NHL or Hodgkin's lymphoma, the incidence of auto-immune adverse events, particularly colitis, was significantly less than previously observed in trials of idelalisib ${ }^{[73]}$. Among the 20 CLL patients on this trial, for which response data were available, 85\% (17) had an objective response to treatment. To date, there have been no reports concerning the mechanisms of resistance to umbralisib, but considering its favourable toxicity profile compared to idelalisib and with on-going trials of umbralisib in combination with other agents, studies detailing umbralisib-refractory disease may emerge.

\section{OTHER BCR-SIGNALLING INHIBITORS}

Inhibitors targeting other components of the BCR signalling pathway have also been or are currently being trialled for CLL. The SYK inhibitor fostamatinib and the dual SYK/Janus kinase 1/3 (JAK1/3) inhibitor cerdulatinib have both been studied. Among 11 patients with relapsed/refractory CLL treated on a phase I/II 
study of fostamatinib the overall response rate was $55 \%$ but the rate of dose-limiting toxicities was high and included diarrhoea, neutropenia and thrombocytopenia ${ }^{[74]}$. In a phase I trial of cerdulatinib that enrolled 8 patients with relapsed/refractory CLL, 2 patients achieved a clinical response ${ }^{[75]}$. Two patients who had previously progressed on therapy with ibrutinib and then progressed while on cerdulatinib were found to harbour the C481 mutation in BTK as well as mutations of TP53 and EP300, which encodes a histone acetyltransferase. A recent in vitro study demonstrated that cerdulatinib may be effective in overcoming ibrutinib resistance and the supportive effects of the tumour microenvironment ${ }^{[76]}$, suggesting that resistance to cerdulatinib in the trial by Hamlin et al ${ }^{[77]}$ may be related to the mutation of TP53 or dysregulation of transcription due to mutation of EP300. Further data are likely to become available when the results of an on-going phase I/IIa trial of cerdulatinib are presented (NCT01994382). Since these trials are comprised of relapsed/refractory and generally heavily pre-treated patients, the response rates are encouraging and suggest that inhibitors targeting alternate components of the BCR-signalling pathway may be effective for some patients with extremely poor risk disease. While it is difficult to draw any conclusions regarding the mechanisms of drug resistance in patients who experienced disease progression in these trials, it is conceivable that the same mechanisms already discussed as being associated with resistance to the BTK and PI3-kinase inhibitors would be relevant to these drugs.

Protein kinase C (PKC) also plays a significant role in BCR signalling and the survival of CLL cell ${ }^{[78]}$. Preclinical studies of enzastaurin suggest that specific inhibition of the PKC-bb isoform may have efficacy in $\mathrm{CLL}^{[79]}$. While there are no reports of clinical trials of enzastaurin in CLL, an on-going phase I trial of MS-553 (NCT03492125), will determine whether the drug is tolerable and whether inhibition of PKC-b may represent another therapeutic strategy for refractory CLL.

\section{INHIBITION OF BCL-2-FAMILY PROTEINS}

CLL cells constitutively overexpress the BCL2 protein. The BCL2 family of proteins comprises proteins that have roles in promoting either cell survival or apoptosis ${ }^{[80]}$. The members of this family with pro-survival functions include BCL2, BCLw, BCLxL, MCL1 and BFL1, while a subset of this group, termed BH3-only proteins, which include BAD, NOXA, BIM, PUMA and tBID, bind to and inhibit pro-survival members of the family. Along with these pro-survival and inhibitory proteins BAX and BAK function as pro-apoptotic effectors by inducing the depolarisation of the outer mitochondrial membrane, resulting in cytochrome $\mathrm{C}$ release and a caspase-dependent apoptotic signalling cascade. Under normal cellular conditions, the balance of proteins in the BCL2 family is tightly regulated, but in several disease conditions, including in CLL, unchecked cell survival and proliferation results from a dramatic shift in this balance. Overexpression of BCL2 in CLL has been linked to low expression of miRs including miR-15a and miR-16-1, which are known to repress BCL2 expression ${ }^{[81]}$. As CLL-cell survival is dependent on overexpression of BCL2, the protein is an attractive target for therapy.

The first drug in a class known as the BH3-mimetics, ABT-263 or navitoclax, was found to be effective in vitro against a range of cell types, including CLL, and in vivo against solid tumours, including small-cell lung carcinoma ${ }^{[82,83]}$. Interestingly, the study by van Delft et al. ${ }^{[82]}$, identified several cell lines and a mouse model of lymphoma that were resistant to navitoclax due to the fact the drug does not inhibit MCL1. In clinical trials, navitoclax had significant antitumour activity but also caused profound thrombocytopaenia due to inhibition of $\mathrm{BCLxL}^{[83]}$. The promising antitumour effects of navitoclax prompted the development of other drugs in this class, most notably venetoclax.

\section{Venetoclax}

Venetoclax (ABT-199) was developed to specifically inhibit BCL2 with only weak activity towards other members of the BCL2 family, including BCLxL thereby minimising thrombocytopaenia as a dose-limiting toxicity ${ }^{[84]}$. In addition to frequent clearing of normal and CLL B cells from the peripheral blood and 
lymph nodes, venetoclax therapy also significantly reduces the number of regulatory $\mathrm{T}$ cells (Tregs) and TNF- $\alpha$ - and IFN- $\lambda$-producing $\mathrm{CD}^{+}$T-cells and significantly increases IFN- $\lambda$ production from NK cells ${ }^{[85]}$. These observations suggest that venetoclax has both direct cytotoxic and immunomodulatory effects. Interestingly, although the venetoclax mechanism of action is TP53-independent ${ }^{[86]}$, patients harbouring TP53 dysfunction have a poorer outcome in clinical trials ${ }^{[87,88]}$.

Despite its efficacy, most heavily pre-treated CLL patients will relapse during or following treatment with venetoclax. Whole-exome sequencing of samples from 8 patients, taken before initiating venetoclax treatment and after the patients developed resistance to the treatment, identified lesions in several cancerrelated genes ${ }^{[89]}$. Mutations of $B T G 1$ and $B R A F$, deletions of $C D N K 2 A / B$ and amplification of PD-L1 were all identified in samples from venetoclax-resistant patients, suggesting there may be multiple mechanisms that confer resistance to venetoclax in CLL. A study by Blombery et al. ${ }^{[00]}$ identified a G101V mutation of BCL2 in samples from 7 of 15 venetoclax-resistant patients. However, the mechanisms that conferred resistance to the drug in the remaining 8 patients in this study were not elucidated. In another recent study, Tausch et al. ${ }^{[91]}$ identified one venetoclax-resistant patient with a D103Y mutation in BCL2, prior to the acquisition of a G101V mutation. In this study 3 of the 4 venetoclax-resistant patients were found to have the G101V mutation. Both studies suggest that the G101V and D103Y mutations interfere with the binding of venetoclax to BCL2. As these mutations were only present after $>2$ years of therapy, it is likely that they were acquired during treatment rather than harboured by the leukemia cells prior to treatment, which is supported by the fact that they were undetectable in all patients prior to commencing treatment. Interestingly, neither mutation of $B C L 2$ was detected in any of the samples from the 8 patients studied by Herling et al ${ }^{[80]}$, providing further evidence that these mutations do not account for every case of venetoclax-resistant CLL.

The notion that alternate pro-survival mechanisms may also play a significant role in conferring resistance to venetoclax is illustrated by preclinical studies of acquired resistance in lymphoma cell lines ${ }^{[92,93]}$. These studies demonstrated that resistance was associated with increased expression of BCLxL and MCL1, an increase in the activity of the PI3-kinase pathway and changes in cellular energy metabolism. These studies also highlight how understanding the mechanisms of resistance can be harnessed to rationalise novel drug combinations; Choudhary et al. ${ }^{[92]}$ demonstrated that venetoclax-resistant NHL cell lines could be sensitised to the $\mathrm{BH} 3$-mimetic by combining the drug with inhibitors of the PI3-kinase signalling pathway. Increased expression of alternate BCL2 family proteins in CLL cells resulting from the interaction of the leukaemic cells with other cell types that comprise the tumour microenvironment is also likely to play an important role in determining the response of patients to venetoclax. This was demonstrated in the study by Vogler et al. ${ }^{[94]}$, which showed that CLL cells co-cultured with stromal cells were 1000-fold less sensitive to ABT-737, a predecessor to venetoclax. It is conceivable that the sensitivity of CLL cells to venetoclax may also be related to changes in the expression of BCL2 and other proteins of the BCL2 family. A preclinical study of venetoclax in multiple myeloma demonstrated that the efficacy of venetoclax is dependent on high expression of BCL2 but also low expression of BCLxL and MCL1 ${ }^{[95]}$. Similar findings were observed in a phase I trial of venetoclax for multiple myeloma; venetoclax was particularly effective for patients with a translocation between chromosomes 11 and 14 (t11:14), which occurs in approximately $20 \%$ of patients and is associated with higher levels of BCL2 expression in relation to other BCL2 family proteins ${ }^{[96]}$.

\section{OVERCOMING OR AVOIDING DRUG RESISTANCE IN CLL}

\section{Combination strategies}

A better understanding of the mechanisms of drug resistance in CLL has highlighted several situations in which targeting two or more signalling pathways may be extremely effective and may avoid the development of drug resistance or disease transformation. In addition, synergy between different drugs may have the potential to reduce the toxicity associated with a single drug without compromising efficacy. This concept has been the focus of numerous preclinical and clinical studies. 
The addition of anti-CD20 antibodies to established therapies has historically been associated with improved clinical efficacy. An initial study combining ibrutinib with rituximab showed promising results in terms of safety and activity for CLL patients with relapsed/refractory disease ${ }^{[97]}$; however, contradictory studies have since shown that antagonism occurs between ibrutinib and anti-CD20 antibodies. This antagonism stems from observations that ibrutinib therapy reduces CXCR4-mediated signalling, resulting in decreased expression of CD20 and impairs T cell function, which reduces the ADCC-mediated effects of rituximab ${ }^{[37,98]}$. Ibrutinib-induced downregulation of $\mathrm{CD} 20$ is reversible, and therefore, the efficacy of this combination treatment might depend on the sequence of administration. This hypothesis is supported by a study in which the response rate to sequential administration of ibrutinib and ofatumumab was $100 \%$ compared to $71 \%$ when the sequence was reversed or $79 \%$ when both drugs were administered simultaneously ${ }^{[99}$. A recent study has shown that ibrutinib and rituximab may be effective in combination when the drugs are given simultaneously when rituximab is administered initially at a small dose but then ramped up over the initial 3 cycles $^{[100]}$. Aside from these considerations regarding drug sequencing, followup studies are required to determine the long-term efficacy of ibrutinib in combination with rituximab. It has been surmised that the efficacy of this regimen may be limited by the fact that both drugs are most active against CLL cells with active BCR signalling and the highest levels of CD20 expression ${ }^{[37]}$.

Studies suggest that patients who develop ibrutinib-resistant disease respond well to venetoclax ${ }^{[101]}$, which raises the possibility that early identification of patients with mutations of BTK or PLC-g2 and intervention with venetoclax may be an effective strategy. However, given the dismal prognosis and lack of effective salvage strategies for patients who develop a Richter's transformation, it would seem logical that strategies that avoid drug resistance and disease transformation would be more effective than salvage therapies. This notion is supported by recent data from clinical trials, including the phase II trial CLARITY of ibrutinib and venetoclax for CLL patients with relapsed/refractory disease ${ }^{[102]}$, which demonstrated that undetectable MRD status was achieved in 53 and $36 \%$ of patients, in blood and bone marrow, respectively. In a similar study by Jain et al. ${ }^{[103]}, 88 \%$ of patients achieved a complete remission (CR) or complete remission with incomplete haematological recovery $(\mathrm{CRi})$, and $61 \%$ were in remission with undetectable MRD in their bone marrow, which is significantly higher than the $6.2 \%-25.8 \% \mathrm{CR}$ rate observed among patients treated with ibrutinib alone ${ }^{[104]}$.

Despite the limitations related to its toxicity, the efficacy of trials of idelalisib demonstrated the therapeutic potential of targeting PI3-kinase in CLL ${ }^{[105]}$. These studies provided a strong rationale for further studies of PI3-kinase inhibitors, including the recently published phase I/Ib trial of the dual PI3-kinase-d and casein kinase-1e (CK-1e) inhibitor umbralisib in combination with the CD20 monoclonal antibody ublituximab in patients with CLL and B-NHL ${ }^{[106]}$. The overall response rate among 22 CLL patients with relapsed/ refractory disease, including 9 (41\%) with deletion of TP53, was $62 \%$. Although these data are encouraging, at the time of analysis, 67 of the $75(89 \%)$ patients on this trial had discontinued therapy, primarily due to disease progression (59\%).

Trials of umbralisib in combination with ibrutinib have also been conducted in CLL and mantle cell lymphoma ${ }^{[55]}$. As already mentioned, the PI3-kinase-d and CK-e inhibitor, umbralisib may represent an alternative, less toxic means of targeting PI3-kinase-mediated signalling compared to idelalisib. In combination with ibrutinib, with or without ublituximab, umbralisib may block the activation of alternate pro-survival signals that arise in CLL cells treated with either drug alone ${ }^{[5,106]}$. Data from the phase I/ Ib trial by Davids et al ${ }^{[5]}$ suggest that this is a well-tolerated regimen with an overall response rate of 90\% among 42 patients with relapsed/refractory CLL or mantle cell lymphoma. Extended follow-up of patients on this trial or additional studies are required to determine the incidence and mechanisms of drug resistance among patients treated with this regimen. 
Trials of venetoclax in combination with CD20-targeted therapies are currently on-going. Although it is unclear whether addition of rituximab has any benefit over venetoclax monotherapy for relapsed/refractory CLL patients ${ }^{[107]}$, the rationale for these trials is supported by preclinical data. A study of venetoclax in combination with rituximab or obinutuzumab demonstrated that the CD20 antibodies countered resistance to venetoclax induced by co-culture of the CLL cells with a stromal layer ${ }^{[108]}$. Previous studies from the same group suggest that this increase in sensitivity is related to the ability of the anti-CD20 antibodies to induce apoptosis-independent cell killing of CLL cells, which has been stimulated by co-culture with stromal cells ${ }^{[109]}$.

\section{Future therapeutic strategies}

Trials of ibrutinib and the next-generation BTK inhibitors demonstrate the potential of targeting this pathway in CLL. A recent study suggests that targeted degradation of BTK may also be a strategy for inactivating this kinase and is effective against BTK harbouring mutations at the C481 residue ${ }^{[110]}$. Dobrovolsky et al. ${ }^{[110]}$ demonstrated that their lead compound, DD-03-171, degrades not only BTK but also the transcription factors IKZF1 and IKZF3, effectively blocking BCR-mediated signalling and inhibiting the proliferation of leukaemic cells from patients with mantle cell lymphoma. The IKZF transcription factors are targets of the immunomodulatory (IMiD) class of drugs and putative driver mutations of IKZF3 occur in approximately $2 \%$ of CLL patients ${ }^{[8]}$.

We and others have demonstrated that paradoxical activation of an alternate pro-survival pathway can occur in CLL cells treated with some inhibitors ${ }^{[70,111,112]}$. In these studies, inhibition of MEK1/2 in the MAPK-ERK1/2 signalling pathway resulted in increased phosphorylation of AKT. These observations raise the possibility that there may be multiple, as yet undefined situations under which crosstalk between pathways may compensate when one pro-survival pathway is blocked and that these mechanisms confer resistance to specific kinase inhibitors. Elucidating the network of crosstalk may help to better understand the mechanisms of drug resistance and identify rational combinations of drugs. This notion is illustrated by our recent studies demonstrating that inhibition of MEK1/2 by binimetinib is effective against CLL cells when combined with an AKT inhibitor ${ }^{[111]}$ or a $\mathrm{BH} 3-$ mimetic $^{[70]}$. The efficacy of similar rational drug combinations has been demonstrated in preclinical studies, including studies showing that inhibition of MCL1 sensitises DLBCL, follicular lymphoma, mantle cell lymphoma ${ }^{[113]}$ and acute myeloid leukaemia ${ }^{[114]}$ cells to venetoclax.

Another therapeutic avenue currently under investigation is inhibition of PD-1, which is involved in immune surveillance of multiple forms of cancer, including CLL. A trial of the PD-1 inhibitor pembrolizumab demonstrated that the drug has activity in patients with a Richter's transformation of $\mathrm{CLL}^{[115]}$. The response of these patients correlated with pre-treatment levels of PD-1 and its ligand PD-L1 in the patients lymph nodes and on the CLL cells, respectively ${ }^{[115]}$. Given that a significant proportion of CLL patients develop a Richter's transformation, the objective response rate of $44 \%$ in this trial is promising and suggests that PD- 1 blockade with pembrolizumab or the PD-1 antibody durvalumab ${ }^{[116]}$ may represent a promising treatment strategy for patients with aggressive disease or disease transformation.

Finally, single drugs that target multiple key signalling pathways may represent an effective means of preventing the evolution of drug resistant CLL clones. Inhibition of the molecular chaperone HSP-90, for example, is known to have significant effects on several key signalling pathways ${ }^{[117]}$. A more targeted approach is illustrated by our studies of the dual PIM and PI3-kinase inhibitor, IBL-202 ${ }^{[118]}$, and by studies of the dual SYK/JAK inhibitor cerdulitinib, which is currently in a clinical trial ${ }^{[75]}$; in vitro studies of both IBL-202 and cerdulitinib ${ }^{[76]}$ demonstrate that these drugs are effective against CLL cells under conditions known to confer resistance to drugs in current clinical use, including ibrutinib and venetoclax. 


\section{CONCLUDING REMARKS}

During the last two decades, there have been significant advances in the therapeutic options for CLL. Although CIT has proven effective, particularly for a subset of patients with markers of good prognosis, the increasing age demographic in many countries means that there is an increasing need for treatment strategies for the elderly, comorbid CLL patients. Targeted therapies are potentially less toxic, may lower secondary malignancy rates and may represent strategies for disease control rather than cure, compared to genotoxic regimens for elderly patients. However, disease relapse and drug resistance are still common among patients treated with BCR-signalling and BCL2 inhibitors, since the development of each novel signalling inhibitor has heralded new mechanisms of drug resistance. A better understanding of these resistance mechanisms should provide the rationale for new strategies to treat refractory disease or to minimise the risk of drug resistance or disease transformation.

\section{DECLARATIONS}

\section{Authors' contributions}

Wrote the first draft of the manuscript: Fatima N, Crassini KR, Best OG

Contributed to revising the manuscript prior to first submission and to addressing the reviewer's comments prior to resubmission: Fatima N, Crassini KR, Thurgood L, Shen Y, Christopherson RI, Kuss B, Mulligan SP, Best OG

\section{Availability of data and materials}

All data sources are referenced in the manuscript and were sourced from online journal repositories.

\section{Financial support and sponsorship}

Thurgood L is supported by a Beat Cancer Early Career Fellowship from the South Australia Cancer Council. All other authors have no financial support or sponsorship to declare.

\section{Conflicts of interest}

All authors declared that there are no conflicts of interest.

\section{Ethical approval and consent to participate}

Not applicable.

\section{Consent for publication}

All authors have read and reviewed the submission.

\section{Copyright}

(c) The Author(s) 2020.

\section{REFERENCES}

1. Chiorazzi N, Rai KR, Ferrarini M. Chronic lymphocytic leukemia. N Engl J Med 2005;352:804-15.

2. Rossi D, Gaidano G. The clinical implications of gene mutations in chronic lymphocytic leukaemia. Br J Cancer 2016;114:849-54.

3. Rossi D, Terzi-di-Bergamo L, De Paoli L, Cerri M, Ghilardi G, et al. Molecular prediction of durable remission after first-line fludarabinecyclophosphamide-rituximab in chronic lymphocytic leukemia. Blood 2015;126:1921-4.

4. Condoluci A, Rossi D. Clonal evolution in chronic lymphocytic leukemia. Clinical Lymphoma Myeloma Leukemia 2019;19:S16-9.

5. Tsimberidou AM, Tam C, Abruzzo LV, O'Brien S, Wierda WG, et al. Chemoimmunotherapy may overcome the adverse prognostic significance of 11q deletion in previously untreated patients with chronic lymphocytic leukemia. Cancer 2009;115:373-80.

6. Stilgenbauer S, Schnaiter A, Paschka P, Zenz T, Rossi M, et al. Gene mutations and treatment outcome in chronic lymphocytic leukemia: results from the CLL8 trial. Blood 2014;123:3247-54.

7. Spunarova M, Tom N, Pavlova S, Mraz M, Brychtova Y, et al. Impact of gene mutations and chromosomal aberrations on progressionfree survival in chronic lymphocytic leukemia patients treated with front-line chemoimmunotherapy: clinical practice experience. Leuk 
Res 2019;81:75-81.

8. Landau DA, Tausch E, Taylor-Weiner AN, Stewart C, Reiter JG, et al. Mutations driving CLL and their evolution in progression and relapse. Nature 2015;526:525-30.

9. Badoux XC, Keating MJ, Wang X, O'Brien SM, Ferrajoli A, et al. Fludarabine, cyclophosphamide, and rituximab chemoimmunotherapy is highly effective treatment for relapsed patients with CLL. Blood 2011;117:3016-24.

10. Hallek M, Fischer K, Fingerle-Rowson G, Fink AM, Busch R, et al. Addition of rituximab to fludarabine and cyclophosphamide in patients with chronic lymphocytic leukaemia: a randomised, open-label, phase 3 trial. Lancet 2010;376:1164-74.

11. Keating MJ, O’Brien S, Albitar M, Lerner S, Plunkett W, et al. Early results of a chemoimmunotherapy regimen of fludarabine, cyclophosphamide, and rituximab as initial therapy for chronic lymphocytic leukemia. J Clin Oncol 2005;23:4079-88.

12. Wierda W, O’Brien S, Wen S, Faderl S, Garcia-Manero G, et al. Chemoimmunotherapy with fludarabine, cyclophosphamide, and rituximab for relapsed and refractory chronic lymphocytic leukemia. J Clin Oncol 2005;23:4070-8.

13. Thompson PA, Tam CS, O’Brien SM, Wierda WG, Stingo F, et al. Fludarabine, cyclophosphamide, and rituximab treatment achieves long-term disease-free survival in IGHV-mutated chronic lymphocytic leukemia. Blood 2016;127:303-9.

14. Benjamini O, Jain P, Trinh L, Qiao W, Strom SS, et al. Second cancers in patients with chronic lymphocytic leukemia who received frontline fludarabine, cyclophosphamide and rituximab therapy: distribution and clinical outcomes. Leuk Lymphoma 2015;56:1643-50.

15. Deans JP, Li H, Polyak MJ. CD20-mediated apoptosis: signalling through lipid rafts. Immunology 2002;107:176-82.

16. Alomari M, Mactier S, Kaufman KL, Best OG, Mulligan SP, et al. Profiling the lipid raft proteome from human MEC1 chronic lymphocytic leukemia cells. J Proteom Bioinform 2014;S7:005.

17. Hammadi M, Youinou P, Tempescul A, Tobón G, Berthou C, et al. Membrane microdomain sphingolipids are required for anti-CD20induced death of chronic lymphocytic leukemia B cells. Haematologica 2012;97:288-96.

18. Fischer K, Bahlo J, Fink AM, Goede V, Herling CD, et al. Long-term remissions after FCR chemoimmunotherapy in previously untreated patients with CLL: updated results of the CLL8 trial. Blood 2016;127:208-15.

19. Brieghel C, Kinalis S, Yde CW, Schmidt AY, Jønson L, et al. Deep targeted sequencing of TP53 in chronic lymphocytic leukemia: clinical impact at diagnosis and at time of treatment. Haematologica 2019;104:789-96.

20. Ferracin M, Zagatti B, Rizzotto L, Cavazzini F, Veronese A, et al. MicroRNAs involvement in fludarabine refractory chronic lymphocytic leukemia. Mol Cancer 2010;9:123.

21. Gagez AL, Duroux-Richard I, Leprêtre S, Orsini-Piocelle F, Letestu R, et al. miR-125b and miR-532-3p predict the efficiency of rituximab-mediated lymphodepletion in chronic lymphocytic leukemia patients. A French Innovative Leukemia Organization study. Haematologica 2017;102:746-54.

22. Cerna K, Oppelt J, Chochola V, Musilova K, Seda V, et al. MicroRNA miR-34a downregulates FOXP1 during DNA damage response to limit BCR signalling in chronic lymphocytic leukaemia B cells. Leukemia 2019;33:403-14.

23. Pozzo F, Bittolo T, Arruga F, Bulian P, Macor P, et al. NOTCH1 mutations associate with low CD20 level in chronic lymphocytic leukemia: evidence for a NOTCH1 mutation-driven epigenetic dysregulation. Leukemia 2016;30:182-9.

24. Tausch E, Beck P, Schlenk RF, Jebaraj BJ, Dolnik A, et al. Prognostic and predictive role of gene mutations in chronic lymphocytic leukemia: results from the pivotal phase III study COMPLEMENT1. Haematologica 2020; Epub ahead of print. doi: 10.3324/ haematol.2019.229161

25. Edelmann J, Tausch E, Landau DA, Robrecht S, Bahlo J, et al. Frequent evolution of copy number alterations in CLL following first-line treatment with FC(R) is enriched with TP53 alterations: results from the CLL8 trial. Leukemia 2017;31:734-8.

26. Baliakas P, Jeromin S, Iskas M, Puiggros A, Plevova K, et al. Cytogenetic complexity in chronic lymphocytic leukemia: definitions, associations, and clinical impact. Blood 2019;133:1205-16.

27. Guièze R, Robbe P, Clifford R, de Guibert S, Pereira B, et al. Presence of multiple recurrent mutations confers poor trial outcome of relapsed/refractory CLL. Blood 2015;126:2110-7.

28. Fischer K, Cramer P, Busch R, Stilgenbauer S, Bahlo J, et al. Bendamustine combined with rituximab in patients with relapsed and/or refractory chronic lymphocytic leukemia: a multicenter phase II trial of the German Chronic Lymphocytic Leukemia Study Group. J Clin Oncol 2011;29:3559-66.

29. Teeling JL, French RR, Cragg MS, van den Brakel J, Pluyter M, et al. Characterization of new human CD20 monoclonal antibodies with potent cytolytic activity against non-Hodgkin lymphomas. Blood 2004;104:1793-800.

30. Bologna L, Gotti E, Da Roit F, Intermesoli T, Rambaldi A, et al. Ofatumumab is more efficient than rituximab in lysing B chronic lymphocytic leukemia cells in whole blood and in combination with chemotherapy. J Immunol 2013;190:231-9.

31. Cartron G, de Guibert S, Dilhuydy MS, Morschhauser F, Leblond V, et al. Obinutuzumab (GA101) in relapsed/refractory chronic lymphocytic leukemia: final data from the phase 1/2 GAUGUIN study. Blood 2014;124:2196-202.

32. Goede V, Fischer K, Busch R, Engelke A, Eichhorst B, et al. Obinutuzumab plus chlorambucil in patients with CLL and coexisting conditions. N Engl J Med 2014;370:1101-10.

33. Ysebaert L, Laprévotte E, Klein C, Quillet-Mary A. Obinutuzumab (GA101) is highly effective against chronic lymphocytic leukemia cells in ex vivo B-cell depletion irrespective of high-risk prognostic markers. Blood Cancer J 2015;5:e367.

34. Burger JA, Chiorazzi N. B cell receptor signaling in chronic lymphocytic leukemia. Trends Immunol 2013;34:592-601.

35. Burger JA, Gribben JG. The microenvironment in chronic lymphocytic leukemia (CLL) and other B cell malignancies: insight into disease biology and new targeted therapies. Semin Cancer Biol 2014;24:71-81.

36. Chen SS, Chang BY, Chang S, Tong T, Ham S, et al. BTK inhibition results in impaired CXCR4 chemokine receptor surface expression, signaling and function in chronic lymphocytic leukemia. Leukemia 2016;30:833-43. 
37. Pavlasova G, Borsky M, Seda V, Cerna K, Osickova J, et al. Ibrutinib inhibits CD20 upregulation on CLL B cells mediated by the CXCR4/SDF-1 axis. Blood 2016;128:1609-13.

38. Herman SEM, Mustafa RZ, Jones J, Wong DH, Farooqui M, et al. Treatment with ibrutinib inhibits BTK- and VLA-4-dependent adhesion of chronic lymphocytic leukemia cells in vivo. Clin Cancer Res 2015;21:4642-51.

39. Kapoor I, Li Y, Sharma A, Zhu H, Bodo J, et al. Resistance to BTK inhibition by ibrutinib can be overcome by preventing FOXO3a nuclear export and PI3K/AKT activation in B-cell lymphoid malignancies. Cell Death Dis 2019;10:924.

40. Pan Z, Scheerens H, Li SJ, Schultz BE, Sprengeler PA, et al. Discovery of selective irreversible inhibitors for Bruton's tyrosine kinase. Chem Med Chem 2007;2:58-61.

41. Burger JA, Tedeschi A, Barr PM, Robak T, Owen C, et al. Ibrutinib as Initial Therapy for Patients with Chronic Lymphocytic Leukemia. N Engl J Med 2015;373:2425-37.

42. Byrd JC, Brown JR, O’Brien S, Barrientos JC, Kay NE, et al. Ibrutinib versus ofatumumab in previously treated chronic lymphoid leukemia. N Engl J Med 2014;371:213-23.

43. Stephens DM, Byrd JC. How I manage ibrutinib intolerance and complications in patients with chronic lymphocytic leukemia. Blood 2019;133:1298-307.

44. Mato AR, Nabhan C, Thompson MC, Lamanna N, Brander DM, et al. Toxicities and outcomes of 616 ibrutinib-treated patients in the United States: a real-world analysis. Haematologica 2018;103:874-9.

45. Furman RR, Cheng S, Lu P, Setty M, Perez AR, et al. Ibrutinib resistance in chronic lymphocytic leukemia. N Engl J Med 2014;370:2352-4.

46. Woyach JA, Furman RR, Liu TM, Ozer HG, Zapatka M, et al. Resistance mechanisms for the Bruton's tyrosine kinase inhibitor ibrutinib. N Engl J Med 2014;370:2286-94.

47. Kanagal-Shamanna R, Jain P, Patel KP, Routbort M, Bueso-Ramos C, et al. Targeted multigene deep sequencing of Bruton tyrosine kinase inhibitor-resistant chronic lymphocytic leukemia with disease progression and Richter transformation. Cancer 2019;125:559-74.

48. Sharma S, Galanina N, Guo A, Lee J, Kadri S, et al. Identification of a structurally novel BTK mutation that drives ibrutinib resistance in CLL. Oncotarget 2016;7:68833-41.

49. Quinquenel A, Fornecker LM, Letestu R, Ysebaert L, Fleury C, et al. Prevalence of BTK and PLCG2 mutations in a real-life CLL cohort still on ibrutinib after 3 years: a FILO group study. Blood 2019;134:641-4.

50. Sinha S, Price-Troska T, Tian S, Secreto CR, Wu X, et al. PD-1 Overexpression in richter's transformation (RT) and aggressive chronic lymphocytic leukemia (CLL) after progression on ibrutinib increases Bcl-2 expression via Akt/mTOR pathway. Am Soc Hematology 2018.

51. Jain N, Havranek O, Singh RK, Khashab T, Shirazi F, et al. Overcoming ibrutinib resistance by targeting phosphatidylinositol-3-kinase signaling in diffuse large B-cell lymphoma. bioRxiv 2019:523761.

52. Del Poeta G, Postorino M, Pozzo F, Del Principe MI, Santinelli E et al. The amount of apoptosis predicts outcome in ibrutinib-treated chronic lymphocytic leukemia (CLL). Blood 2018;132 (Supplement 1):4397.

53. Tissino E, Benedetti D, Herman SEM, Ten Hacken E, Ahn IE, et al. Functional and clinical relevance of VLA-4 (CD49d/CD29) in ibrutinib-treated chronic lymphocytic leukemia. J Exp Med 2018;215:681-97.

54. Hillmen P, Rawstron A, Brock K, Munoz Vicente S, Yates F, et al. Ibrutinib plus venetoclax in relapsed/refractory CLL: results of the bloodwise TAP clarity study. Blood 2018;132 (Supplement 1):182.

55. Davids MS, Kim HT, Nicotra A, Savell A, Francoeur K, et al.; Blood Cancer Research Partnership of the Leukemia and Lymphoma Society. Umbralisib in combination with ibrutinib in patients with relapsed or refractory chronic lymphocytic leukaemia or mantle cell lymphoma: a multicentre phase 1-1b study. Lancet Haematol 2019;6:e38-47.

56. Wang M, Rule S, Zinzani PL, Goy A, Casasnovas O, et al. Acalabrutinib in relapsed or refractory mantle cell lymphoma (ACE-LY-004): a single-arm, multicentre, phase 2 trial. Lancet 2018;391:659-67.

57. Tam C, Grigg AP, Opat S, Ku M, Gilbertson M, et al. The BTK inhibitor, Bgb-3111, is safe, tolerable, and highly active in patients with relapsed/refractory B-cell malignancies: initial report of a phase 1 first-in-human trial. Blood 2015;126.

58. Walter HS, Rule SA, Dyer MJ, Karlin L, Jones C, et al. A phase 1 clinical trial of the selective BTK inhibitor ONO/GS-4059 in relapsed and refractory mature B-cell malignancies. Blood 2016;127:411-9.

59. Montalban X, Arnold DL, Weber MS, Staikov I, Piasecka-Stryczynska K, et al.; Evobrutinib Phase 2 Study Group. Placebo-Controlled Trial of an Oral BTK Inhibitor in Multiple Sclerosis. N Engl J Med 2019;380:2406-17.

60. Goess C, Harris CM, Murdock S, McCarthy RW, Sampson E, et al. ABBV-105, a selective and irreversible inhibitor of Bruton's tyrosine kinase, is efficacious in multiple preclinical models of inflammation. Mod Rheumatol 2019;29:510-22.

61. Park JK, Byun JY, Park JA, Kim YY, Lee YJ, et al. HM71224, a novel Bruton's tyrosine kinase inhibitor, suppresses B cell and monocyte activation and ameliorates arthritis in a mouse model: a potential drug for rheumatoid arthritis. Arthritis Res Ther 2016;18:91.

62. Liclican A, Serafini L, Xing W, Czerwieniec G, Steiner B, et al. Biochemical characterization of tirabrutinib and other irreversible inhibitors of Bruton's tyrosine kinase reveals differences in on - and off - target inhibition. Biochim Biophys Acta Gen Subj 2020;1864:129531.

63. Brown JR, Barrientos JC, Barr PM, Flinn IW, Burger JA, et al. The Bruton tyrosine kinase inhibitor ibrutinib with chemoimmunotherapy in patients with chronic lymphocytic leukemia. Blood 2015;125:2915-22.

64. Lampson BL, Brown JR. Are BTK and PLCG2 mutations necessary and sufficient for ibrutinib resistance in chronic lymphocytic leukemia? Expert Rev Hematol 2018;11:185-94.

65. Brown JR, Byrd JC, Coutre SE, Benson DM, Flinn IW, et al. Idelalisib, an inhibitor of phosphatidylinositol 3-kinase p1108, for relapsed/ refractory chronic lymphocytic leukemia. Blood 2014;123:3390-7. 
66. Lampson BL, Kasar SN, Matos TR, Morgan EA, Rassenti L, et al. Idelalisib given front-line for treatment of chronic lymphocytic leukemia causes frequent immune-mediated hepatotoxicity. Blood 2016;128:195-203.

67. Woyach JA, Johnson AJ. Targeted therapies in CLL: mechanisms of resistance and strategies for management. Blood 2015;126:471-7.

68. Scheffold A, Jebaraj BMC, Tausch E, Bloehdorn J, Ghia P, et al. IGF1R as druggable target mediating PI3K- $\delta$ inhibitor resistance in a murine model of chronic lymphocytic leukemia. Blood 2019;134:534-47.

69. Murali I, Kasar S, McWilliams EM, Itchaki G, Tyekucheva S, et al. Activating MAPK pathway mutations mediate primary resistance to PI3K inhibitors in chronic lymphocytic leukemia (CLL). Am Soc Hematology 2018.

70. Crassini K, Shen Y, Stevenson WS, Christopherson R, Ward C, et al. MEK1/2 inhibition by binimetinib is effective as a single agent and potentiates the actions of Venetoclax and ABT-737 under conditions that mimic the chronic lymphocytic leukaemia (CLL) tumour microenvironment. Br J Haematol 2018;182:360-72.

71. Flinn IW, Hillmen P, Montillo M, Nagy Z, Illés Á, et al. The phase 3 DUO trial: duvelisib vs ofatumumab in relapsed and refractory CLL/ SLL. Blood 2018;132:2446-55.

72. Kim JH, Kim WS, Park C. Interleukin-6 mediates resistance to PI3K-pathway-targeted therapy in lymphoma. BMC Cancer 2019;19:936.

73. Burris HA, Flinn IW, Patel MR, Fenske TS, Deng C, et al. Umbralisib, a novel PI3K $\delta$ and casein kinase- $1 \varepsilon$ inhibitor, in relapsed or refractory chronic lymphocytic leukaemia and lymphoma: an open-label, phase 1, dose-escalation, first-in-human study. Lancet Oncol 2018;19:486-96.

74. Friedberg JW, Sharman J, Sweetenham J, Johnston PB, Vose JM, et al. Inhibition of Syk with fostamatinib disodium has significant clinical activity in non-Hodgkin lymphoma and chronic lymphocytic leukemia. Blood 2010;115:2578-85.

75. Coffey GP, Feng J, Betz A, Pandey A, Birrell M, et al. Cerdulatinib Pharmacodynamics and Relationships to Tumor Response Following Oral Dosing in Patients with Relapsed/Refractory B-cell Malignancies. Clin Cancer Res 2019;25:1174-84.

76. Guo A, Lu P, Coffey G, Conley P, Pandey A, et al. Dual SYK/JAK inhibition overcomes ibrutinib resistance in chronic lymphocytic leukemia: Cerdulatinib, but not ibrutinib, induces apoptosis of tumor cells protected by the microenvironment. Oncotarget 2017;8:12953-67.

77. Hamlin PA, Farber CM, Fenske TS, Khatcheressian JL, Miller CB, et al. The dual SYK/JAK inhibitor cerdulatinib demonstrates rapid tumor responses in a phase 2 study in patients with relapsed/refractory B-and T-cell non-Hodgkin lymphoma (NHL). Hematological Oncol 2017;35:74.

78. Abrams ST, Lakum T, Lin K, Jones GM, Treweeke AT, et al. B-cell receptor signaling in chronic lymphocytic leukemia cells is regulated by overexpressed active protein kinase CbetaII. Blood 2007;109:1193-201.

79. Nakagawa R, Vukovic M, Tarafdar A, Cosimo E, Dunn K, et al. Generation of a poor prognostic chronic lymphocytic leukemia-like disease model: PKC $\alpha$ subversion induces up-regulation of PKC $\beta I I$ expression in B lymphocytes. Haematologica 2015;100:499-510.

80. Burlacu A. Regulation of apoptosis by Bcl-2 family proteins. J Cell Mol Med 2003;7:249-57.

81. Cimmino A, Calin GA, Fabbri M, Iorio MV, Ferracin M, et al. miR-15 and miR-16 induce apoptosis by targeting BCL2. Proc Natl Acad Sci U S A 2005;102:13944-9.

82. van Delft MF, Wei AH, Mason KD, Vandenberg CJ, Chen L, et al. The BH3 mimetic ABT-737 targets selective Bcl-2 proteins and efficiently induces apoptosis via Bak/Bax if Mcl-1 is neutralized. Cancer Cell 2006;10:389-99.

83. Gandhi L, Camidge DR, Ribeiro de Oliveira M, Bonomi P, Gandara D, et al. Phase I study of Navitoclax (ABT-263), a novel Bcl-2 family inhibitor, in patients with small-cell lung cancer and other solid tumors. J Clin Oncol 2011;29:909-16.

84. Delbridge AR, Strasser A. The BCL-2 protein family, BH3-mimetics and cancer therapy. Cell Death Differ 2015;22:1071-80.

85. de Weerdt I, Hofland T, Dobber J, Dubois J, Eldering E, et al. First Evidence of Restoration of T and NK Cell Compartment after Venetoclax Treatment. Blood 2018;132 (Supplement 1):1860.

86. Anderson MA, Deng J, Seymour JF, Tam C, Kim SY, et al. The BCL2 selective inhibitor venetoclax induces rapid onset apoptosis of CLL cells in patients via a TP53-independent mechanism. Blood 2016;127:3215-24.

87. Kater AP, Seymour JF, Hillmen P, Eichhorst B, Langerak AW, et al. Fixed duration of venetoclax-rituximab in relapsed/refractory chronic lymphocytic leukemia eradicates minimal residual disease and prolongs survival: post-treatment follow-up of the MURANO phase III study. J Clin Oncol 2019;37:269-77.

88. Roberts AW, Davids MS, Pagel JM, Kahl BS, Puvvada SD, et al. Targeting BCL2 with Venetoclax in Relapsed Chronic Lymphocytic Leukemia. N Engl J Med 2016;374:311-22.

89. Herling CD, Abedpour N, Weiss J, Schmitt A, Jachimowicz RD, et al. Clonal dynamics towards the development of venetoclax resistance in chronic lymphocytic leukemia. Nat Commun 2018;9:727.

90. Blombery P, Anderson MA, Gong JN, Thijssen R, Birkinshaw RW, et al. Acquisition of the recurrent Gly101Val mutation in BCL2 confers resistance to venetoclax in patients with progressive chronic lymphocytic leukemia. Cancer Discov 2019;9:342-53.

91. Tausch E, Close W, Dolnik A, Bloehdorn J, Chyla B, et al. Venetoclax resistance and acquired BCL2 mutations in chronic lymphocytic leukemia. Haematologica 2019;104:e434-7.

92. Choudhary GS, Al-Harbi S, Mazumder S, Hill BT, Smith MR, et al. MCL-1 and BCL-xL-dependent resistance to the BCL-2 inhibitor ABT-199 can be overcome by preventing PI3K/AKT/mTOR activation in lymphoid malignancies. Cell Death Dis 2015;6:e1593.

93. Guièze R, Liu VM, Rosebrock D, Jourdain AA, Hernández-Sánchez M, et al. Mitochondrial reprogramming underlies resistance to BCL2 inhibition in lymphoid malignancies. Cancer Cell 2019;36:369-84.e13.

94. Vogler M, Butterworth M, Majid A, Walewska RJ, Sun XM, et al. Concurrent up-regulation of BCL-XL and BCL2A1 induces approximately 1000-fold resistance to ABT-737 in chronic lymphocytic leukemia. Blood 2009;113:4403-13.

95. Punnoose EA, Leverson JD, Peale F, Boghaert ER, Belmont LD, et al. Expression profile of BCL-2, BCL-XL, and MCL-1 predicts pharmacological response to the BCL-2 selective antagonist venetoclax in multiple myeloma models. Mol Cancer Ther 2016;15:1132-44. 
96. Touzeau C, Dousset C, Le Gouill S, Sampath D, Leverson JD, et al. The Bcl-2 specific BH3 mimetic ABT-199: a promising targeted therapy for $\mathrm{t}(11 ; 14)$ multiple myeloma. Leukemia 2014;28:210-2.

97. Burger JA, Keating MJ, Wierda WG, Hartmann E, Hoellenriegel J, et al. Safety and activity of ibrutinib plus rituximab for patients with high-risk chronic lymphocytic leukaemia: a single-arm, phase 2 study. Lancet Oncol 2014;15:1090-9.

98. Skarzynski M, Niemann CU, Lee YS, Martyr S, Maric I, et al. Interactions between ibrutinib and anti-CD20 antibodies: competing effects on the outcome of combination therapy. Clin Cancer Res 2016;22:86-95.

99. Jaglowski SM, Jones JA, Nagar V, Flynn JM, Andritsos LA, et al. Safety and activity of BTK inhibitor ibrutinib combined with ofatumumab in chronic lymphocytic leukemia: a phase 1b/2 study. Blood 2015;126:842-50.

100. Shanafelt TD, Wang XV, Kay NE, Hanson CA, O’Brien S, et al. Ibrutinib-rituximab or chemoimmunotherapy for chronic lymphocytic leukemia. N Engl J Med 2019;381:432-43.

101. Jones JA, Mato AR, Wierda WG, Davids MS, Choi M, et al. Venetoclax for chronic lymphocytic leukaemia progressing after ibrutinib: an interim analysis of a multicentre, open-label, phase 2 trial. Lancet Oncol 2018;19:65-75.

102. Hillmen P, Rawstron AC, Brock K, Muñoz-Vicente S, Yates FJ, et al. Ibrutinib plus venetoclax in relapsed/refractory chronic lymphocytic leukemia: the CLARITY study. J Clin Oncol 2019;37:2722-9.

103. Jain N, Keating M, Thompson P, Ferrajoli A, Burger J, et al. Ibrutinib and Venetoclax for First-Line Treatment of CLL. N Engl J Med 2019;380:2095-103.

104. O'Brien S, Furman RR, Coutre S, Flinn IW, Burger JA, et al. Single-agent ibrutinib in treatment-naïve and relapsed/refractory chronic lymphocytic leukemia: a 5-year experience. Blood 2018;131:1910-9.

105. Fruman DA, Cantley LC. Idelalisib--a PI3K $\delta$ inhibitor for B-cell cancers. N Engl J Med 2014;370:1061-2.

106. Lunning M, Vose J, Nastoupil L, Fowler N, Burger JA, et al. Ublituximab and umbralisib in relapsed/refractory B-cell non-Hodgkin lymphoma and chronic lymphocytic leukemia. Blood 2019;134:1811-20.

107. Mato AR, Roeker LE, Eyre TA, Nabhan C, Lamanna N, et al. A retrospective comparison of venetoclax alone or in combination with an anti-CD20 monoclonal antibody in R/R CLL. Blood Adv 2019;3:1568-73.

108. Thijssen R, Slinger E, Weller K, Geest CR, Beaumont T, et al. Resistance to ABT-199 induced by microenvironmental signals in chronic lymphocytic leukemia can be counteracted by CD20 antibodies or kinase inhibitors. Haematologica 2015;100:e302-6.

109. Jak M, van Bochove GG, van Lier RA, Eldering E, van Oers MH. CD40 stimulation sensitizes CLL cells to rituximab-induced cell death. Leukemia 2011;25:968-78.

110. Dobrovolsky D, Wang ES, Morrow S, Leahy C, Faust T, et al. Bruton tyrosine kinase degradation as a therapeutic strategy for cancer. Blood 2019;133:952-61.

111. Shen Y, Crassini K, Sandhu S, Fatima N, Christopherson RI, et al. Dual inhibition of MEK1/2 and AKT by binimetinib and MK2206 induces apoptosis of chronic lymphocytic leukemia cells under conditions that mimic the tumor microenvironment. Leuk Lymphoma 2019;60:1632-43.

112. Shen Y, Crassini KR, O’Dwyer ME, O’Neill MF, Christopherson R, et al. The dual PI3/PIM-kinase inhibitor, Ibl-202, is highly synergistic with venetoclax against CLL cells, and TP53-knock-out cells, and under conditions that mimic the tumor microenvironment. Blood 2018;132(Supplement 1):1870.

113. Tahir SK, Smith ML, Hessler P, Rapp LR, Idler KB, et al. Potential mechanisms of resistance to venetoclax and strategies to circumvent it. BMC Cancer 2017;17:399.

114. Ramsey HE, Fischer MA, Lee T, Gorska AE, Arrate MP, et al. A novel MCL1 inhibitor combined with venetoclax rescues venetoclaxresistant acute myelogenous leukemia. Cancer Discov 2018;8:1566-81.

115. Ding W, LaPlant BR, Call TG, Parikh SA, Leis JF, et al. Pembrolizumab in patients with CLL and Richter transformation or with relapsed CLL. Blood 2017;129:3419-27.

116. Herrera AF, Goy A, Mehta A, Ramchandren R, Pagel JM, et al. Safety and activity of ibrutinib in combination with durvalumab in patients with relapsed or refractory follicular lymphoma or diffuse large B-cell lymphoma. Am J Hematol 2020;95:18-27.

117. Gao C, Peng YN, Wang HZ, Fang SL, Zhang M, et al. Inhibition of heat shock protein 90 as a novel platform for the treatment of cancer. Curr Pharm Des 2019;25:849-55.

118. Crassini K, Shen Y, O’Dwyer M, O’Neill M, Christopherson R, et al. The dual inhibitor of the phosphoinositol-3 and PIM kinases, IBL202 , is effective against chronic lymphocytic leukaemia cells under conditions that mimic the hypoxic tumour microenvironment. Br J Haematol 2018;182:654-69.

119. Ljungström V, Cortese D, Young E, Pandzic T, Mansouri L, et al. Whole-exome sequencing in relapsing chronic lymphocytic leukemia: clinical impact of recurrent RPS15 mutations. Blood 2016;127:1007-16.

120. Chai-Adisaksopha C, Brown JR. FCR achieves long-term durable remissions in patients with IGHV-mutated CLL. Blood 2017;130:2278-82.

121. Fischer K, Cramer P, Busch R, Böttcher S, Bahlo J, et al. Bendamustine in combination with rituximab for previously untreated patients with chronic lymphocytic leukemia: a multicenter phase II trial of the German Chronic Lymphocytic Leukemia Study Group. J Clin Oncol 2012;30:3209-16.

122. Eichhorst B, Fink AM, Bahlo J, Busch R, Kovacs G, et al. First-line chemoimmunotherapy with bendamustine and rituximab versus fludarabine, cyclophosphamide, and rituximab in patients with advanced chronic lymphocytic leukaemia (CLL10): an international, openlabel, randomised, phase 3, non-inferiority trial. Lancet Oncol 2016;17:928-42.

123. Burger JA, Landau DA, Taylor-Weiner A, Bozic I, Zhang H, et al., Clonal evolution in patients with chronic lymphocytic leukaemia developing resistance to BTK inhibition. Nat Commun 2016;7:11589. 